\title{
Out of focus holography at Effelsberg
}

\author{
Uwe Bach* \\ Max-Planck-Institut fuer Radioastrononmie, Bonn, Germany \\ E-mail: ubach@mpifr.de
}

\begin{abstract}
Recently a holography technique was developed which allows to obtain low resolution maps of the wavefront-errors in an antenna surface using astronomical observations. This "out-of-focus (OOF)" holography requires - in contrast to traditional holography measurements - only several focused and out of focus images of a compact source at a good signal to noise ratio. The aperture is then parameterized by Zernike polynomials that describe different modes of surface errors. The obvious advantage is that this technique can be applied over a wide range of elevations. Motivate by this study, the OOF technique was successfully tested at the $100 \mathrm{~m}$ Effelsberg telescope using the $32 \mathrm{GHz}$ multi-horn receiver in the secondary focus. We report on the implementation of the OOF at Effelsberg and the observations, data reduction and analysis of the OOF maps. The final aim is to further improve the correction table that is used for the active surface of the Effelsberg sub-reflector and therewith reduce the elevation dependent gain loss.
\end{abstract}

12th European VLBI Network Symposium and Users Meeting

7-10 October 2014

Cagliari, Italy

\footnotetext{
* Speaker.
} 


\section{Introduction}

Traditional holography methods require beam maps with very high signal to noise ratios that are usually only provide by terrestrial transmitters or the beacon signals from geostationary satellites. To observe those strong signals with astronomical radio antennas requires the use of special receivers and backends. Over the years several holography measurements using geostationary satellites were done at Effelsberg. Those campaigns usually lasted a day or two and the data reduction was time consuming as well. Therewith the surface rms of the main reflector was reduced to only $0.55 \mathrm{~mm}$ at an elevation of $32^{\circ}$, which is the elevation of the satellite. The gain elevation dependence of the Effelsberg antenna therefore peaks at around $30^{\circ}$ to $40^{\circ}$ elevation and falls off towards higher and lower elevations.

In 2007 a method was developed to obtain low-resolution maps of the wavefront-errors in an antenna surface using astronomical sources and detectors $([1,2]$. The technique requires to measure a series of beam maps at perfect focus and two positive and negatively defocused images of a compact source at a good signal to noise ratio. To reduce the number of free parameters the antenna surface is described by Zernike polynomials that describe different modes of surface errors. From those theoretical beam maps are calculated and are compared with the measured beam maps. Since this method is based on the amplitudes of the beam pattern only a single image would not allow to fully solve the coefficients of the Zernike polynomials unambiguously, but by introducing a known phase shift due to the two defocus images the coefficients can be determined. The obvious advantage of this technique is that it uses the normal astronomical receivers, observing modes that are implemented and can be applied over a wide range of elevations. Therefore this method caught our attention and we tested the OOF holography at Effelsberg.

\section{The Effelsberg Antenna}

The support structure of the Effelsberg main dish has been constructed according to the socalled homologous principle. This means that at all elevation angles, the surface always forms a paraboloid, though with variation in the focal length. However, there are known deviations from the parabolic shape that are described in a finite element model. Therefore, when in 2006 the Effelsberg antenna was equipped with a new $6.5 \mathrm{~m}$ diameter sub-reflector with more accurate panels, also an active surface control was included. The new sub-reflector consists of 96 individual panels with actuators which are adjusted for each elevation via a look-up table based on the finite element model. Hence, not only the peak efficiency increased, but also the gain elevation dependence for secondary focus observations became significantly flatter ([3]. The OOF technique appears to be a suitable method to verify the finite element model predictions or it might be even possible to further improve the table.

\section{OOF holography at Effelsberg}

On-the-fly maps are commonly used to observe extended structures with the Effelsberg antenna and the observing mode is generally implemented in the control system ${ }^{1}$. For the OOF

\footnotetext{
${ }^{1}$ Effelsberg User Guide: http://www.eff100mwiki.mpifr-bonn.mpg.de
} 


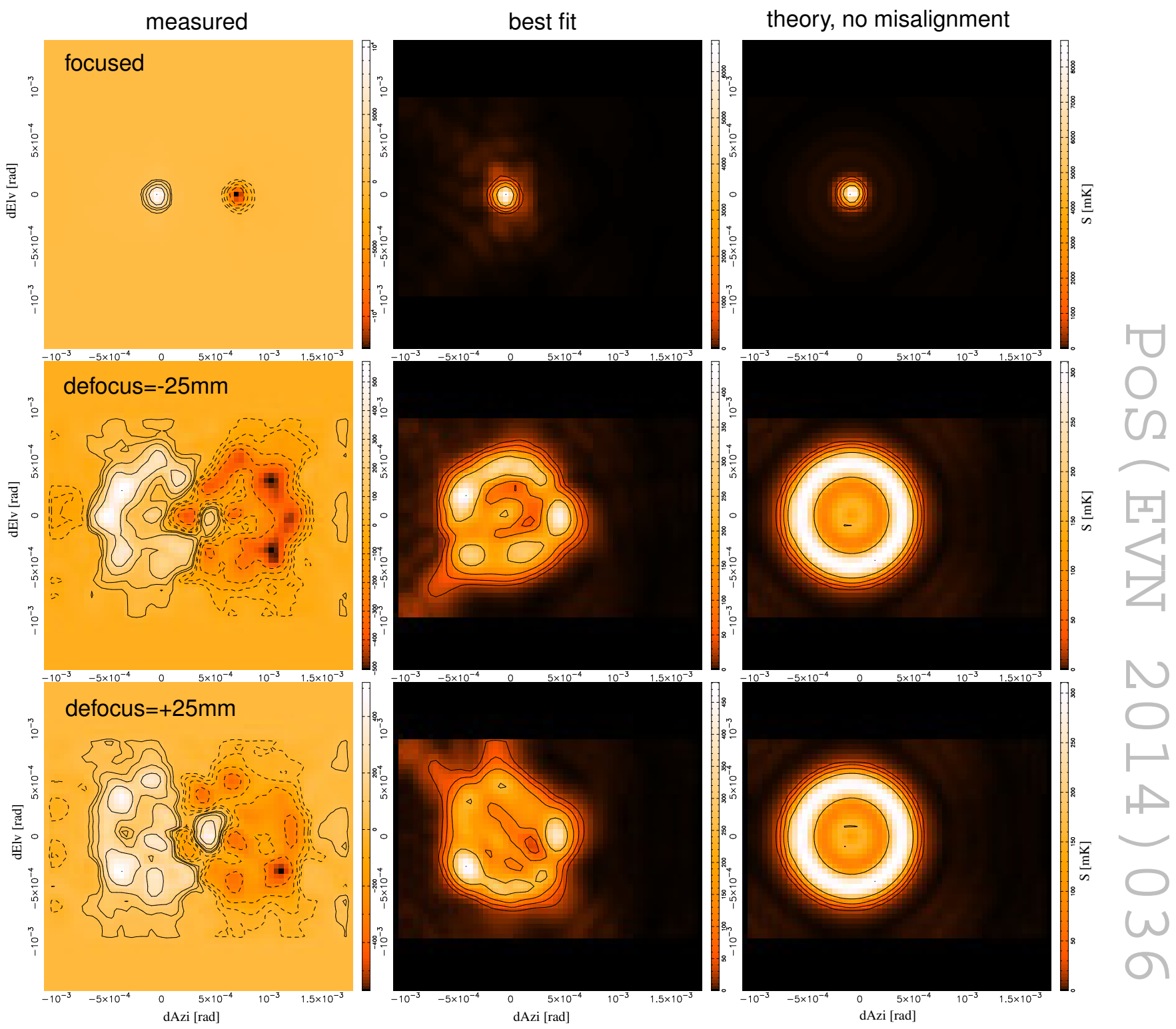

Figure 1: Set of OOF images and best-fits from the OOF software. Top to bottom: Images at perfect focus and defocused by $-25 \mathrm{~mm}$ and $+25 \mathrm{~mm}$, respectively. Left column: Measured beam maps of $550 \times$ $360 \mathrm{arcsec}$ in azimuth and elevation direction. The beam size of the focused image is 26 arcsec and the target was 3C84 with a flux density of about $24 \mathrm{Jy}$. The RMS in the map is about $21 \mathrm{mJy}$ resulting in an SNR of about $\sim 1100$. Middle column: Best-fit images that were obtained by the OOF software. Right column: For comparison the expected perfect image, assuming no optical errors.

observations the multi-beam receiver at $32 \mathrm{GHz}$ was chosen, as a good compromise in terms of sensitivity, resolution and weather dependence. The $32 \mathrm{GHz}$ receiver has a typical system temperature of $55 \mathrm{~K}$, a beam size of 26 arcsec and provides enough signal to noise for OOF observations using sources of about $7-8 \mathrm{Jy}$ and more.

The two outer horns providing left circular polarization were used to measure maps of $550 \times$ 360 arcsec in azimuth and elevation, corresponding to 37 on-the-fly azimuth scans with 10 arcsec 

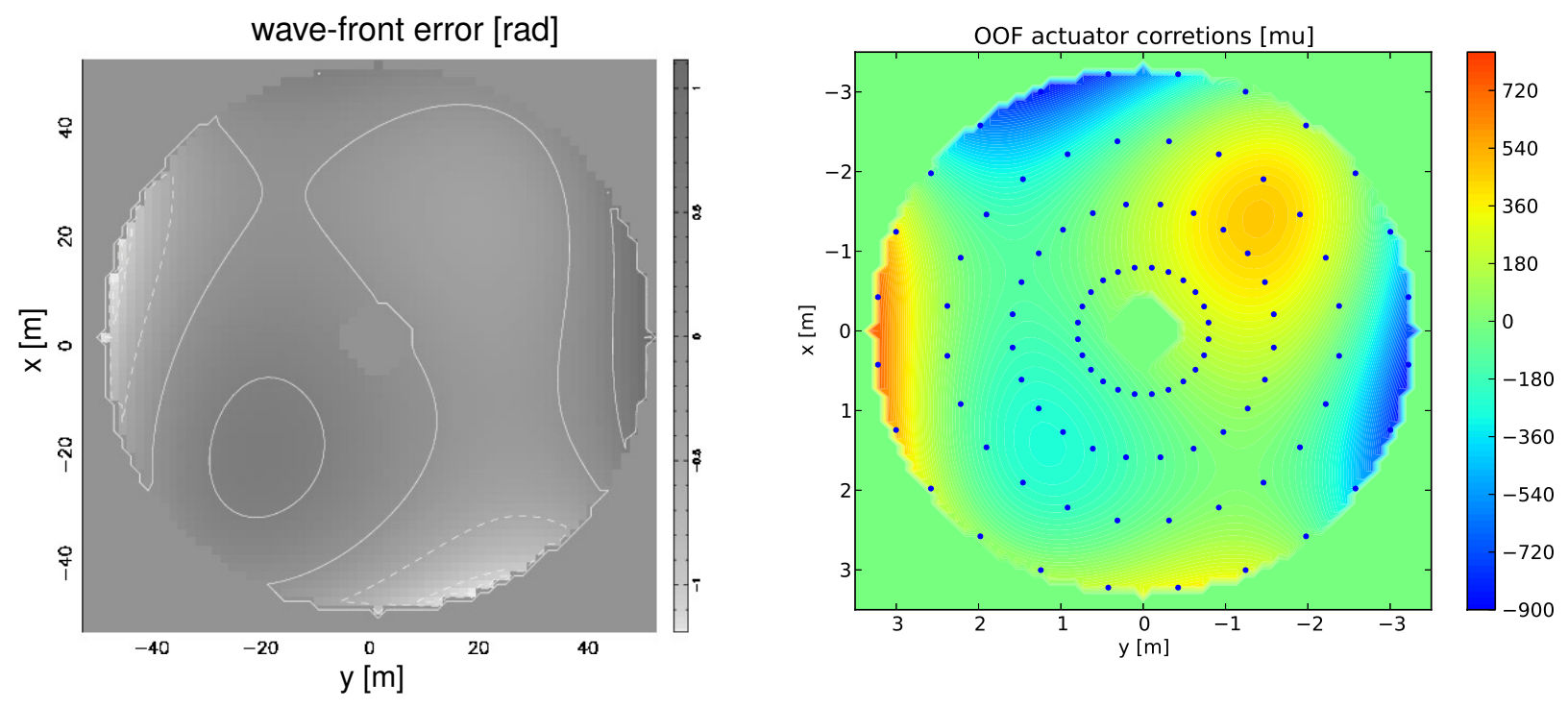

Figure 2: Left panel: The resulting wave-front error map calculated by the OOF software. The x-y range corresponds to the size of the main dish. This map was observed at $\sim 30^{\circ}$ elevation, where Effelsberg has its peak efficiency. Indeed the errors are rather small and only the edges show some larger errors (see text for more details). Right panel: The wave-front errors scaled to the size of the sub-reflector and converted to $\mu \mathrm{m}$. At $32 \mathrm{GHz}$ ( $9.3 \mathrm{~mm}$ wavelength) one rad of phase-error in the wave-front corresponds to $0.71 \mathrm{~mm}$ of displacement of the surface. The blues dots mark the actuator positions. Note that the axis of both panels are mirrored with respect to the centre.

separation. The maps need to be sufficiently large to cover the extended defocused beam images of both horns. At a velocity of $40 \mathrm{arcsec} / \mathrm{s}$ a full map takes about 13 to 14 minutes and therefore a series of three out of focus maps about 45 minutes. Example images are shown in the first column of Figure 1.

After some preprocessing of all three maps in the Effelsberg mapping software, e.g. calculation of antenna temperature (using a noise diode) and differencing of the two horns to reduce weather effects (beam switched images), the maps are written as times series data of the positions in azimuth and elevation and antenna temperature into three tables of a single fits-file. The time series data is then processed with the OOF software ${ }^{2}$ of B. Nikolic. The details of the OOF analysis and algorithm to infer the Zernike coefficients are described in [1]. The only customization of the software that was done at Effelsberg is the introduction of our antenna parameters.

Several test were performed to measure the reliability of the software. Measurements were done at different elevations with the active surface turned on and off and lateral offset of the focus were introduced to produce misalignments by intention. An example for an OOF measurement at $\sim 30^{\circ}$ elevation is shown in Figure 2. Since this was measured at an elevation where the antenna is supposed to have its best performance, the wave-front errors are indeed small. Only at the edges some larger deviations are visible which are likely a combination of two effects: the known misalignment of the outer perforate panels of the main dish and the reduced SNR because of the horn tapering. At $32 \mathrm{GHz}$ (9.3 mm wavelength) one rad of phase-error in the wave-front corresponds to

\footnotetext{
${ }^{2}$ http://www.mrao.cam.ac.uk/ bn204/oof/
} 

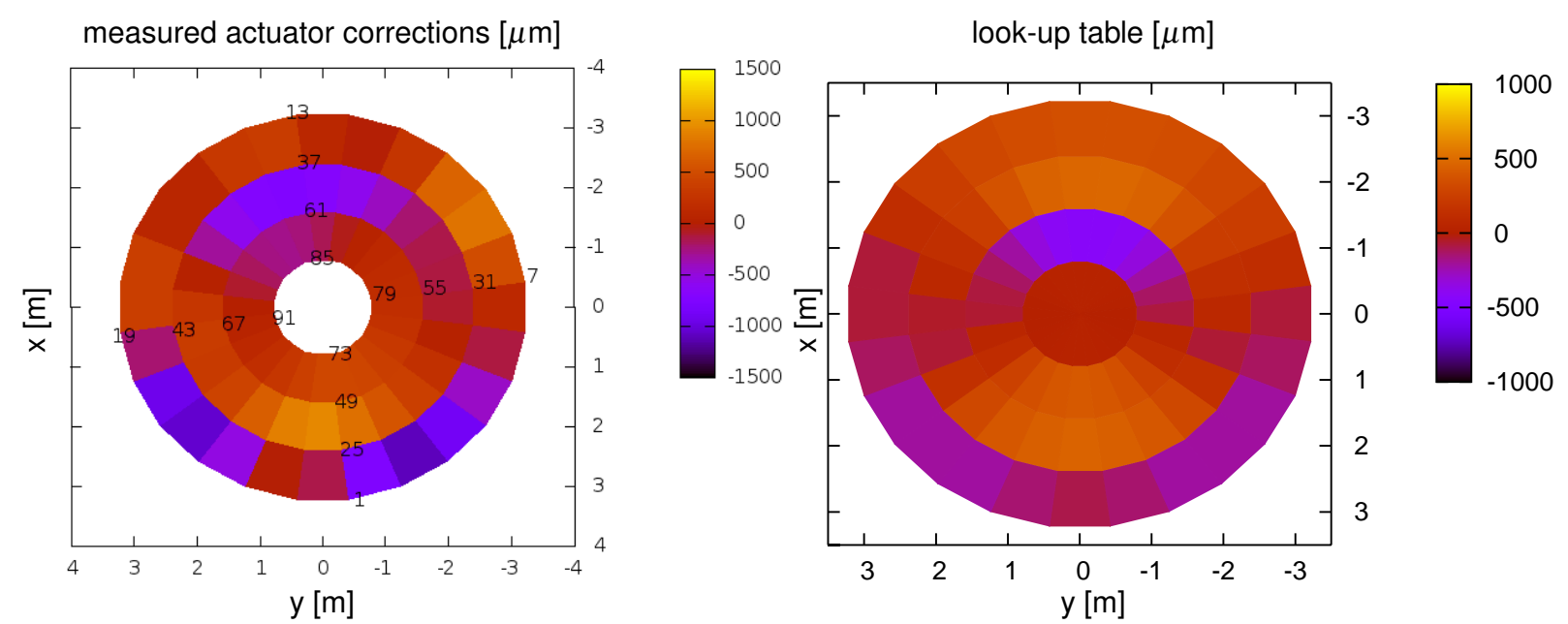

Figure 3: Left panel: Map of actuator correction calculated from the results of an OOF measurement at $\sim 70^{\circ}$ elevation. The numbers denote the actuator labels in the look-up table. Right panel: Illustration of the corresponding entries from the sub-reflector look-up table for $70^{\circ}$ elevation. In general the agreement between the OOF measurement and the finite element model is quit good, but also some deviations are visible (see text).

$0.71 \mathrm{~mm}$ of displacement of the surface. The right panel in Fig. 2 shows phase-errors converted to $\mu \mathrm{m}$ and scaled to the sub-reflector with an overlay of the actuator positions (blue dots). From these maps correction tables for the sub-reflector look-up table can be calculated.

Another OOF measurement was done at higher elevations where the deviations from the parabolic shape are supposed to be larger. For those measurements the active surface was turned off, so that the resulting images should reflect the correction normally introduced by the look-up table. A comparison between the calculated actuator correction and the actual positions of the actuator from the look-up table are shown in Figure 3. The images agree in general quite well and also the amount of correction is well estimated by the OOF measurement. The shape of the deformation is dominated by a second order coma effect. Only in the upper part of the plot the finite element model predicts that the negative part should appear in the first ring only, but the measurements suggest it to be dominant in the second ring. If that is true has to be verified by repeated measurements and possibly modifications of the look-up table.

To unambiguously measure the orientation of the images and the sign of the wave-front error a set of six panels (actuators: 4, 5, 6, 28, 29, 30, 52, 53, 54, 76, 77, and 78) was lifted by $0.5 \mathrm{~mm}$ resulting in a portion of the sub-reflector that was higher than the rest (Fig. 4). Although in the actual wave-front error map (Fig. 4, left panel) the misalignment is only visible as a fuzzy blob, the lifted panels are clearly visible when the wave-front error map is translated to corrections on a panel basis. The lifted panels are marked between solid lines in the lower right of the plot (Fig. 4, right panel). The measured shift agrees well with the introduced error.

In conclusion the tests of the OOF technique at Effelsberg can be regarded as successful and have shown that meaningful results can be obtained. Due to scheduling and weather constraints an actual improvement of the look-up table could not be obtained yet. However, more observations are planed and we are looking forward that the OOF technique will help to improve the corrections 

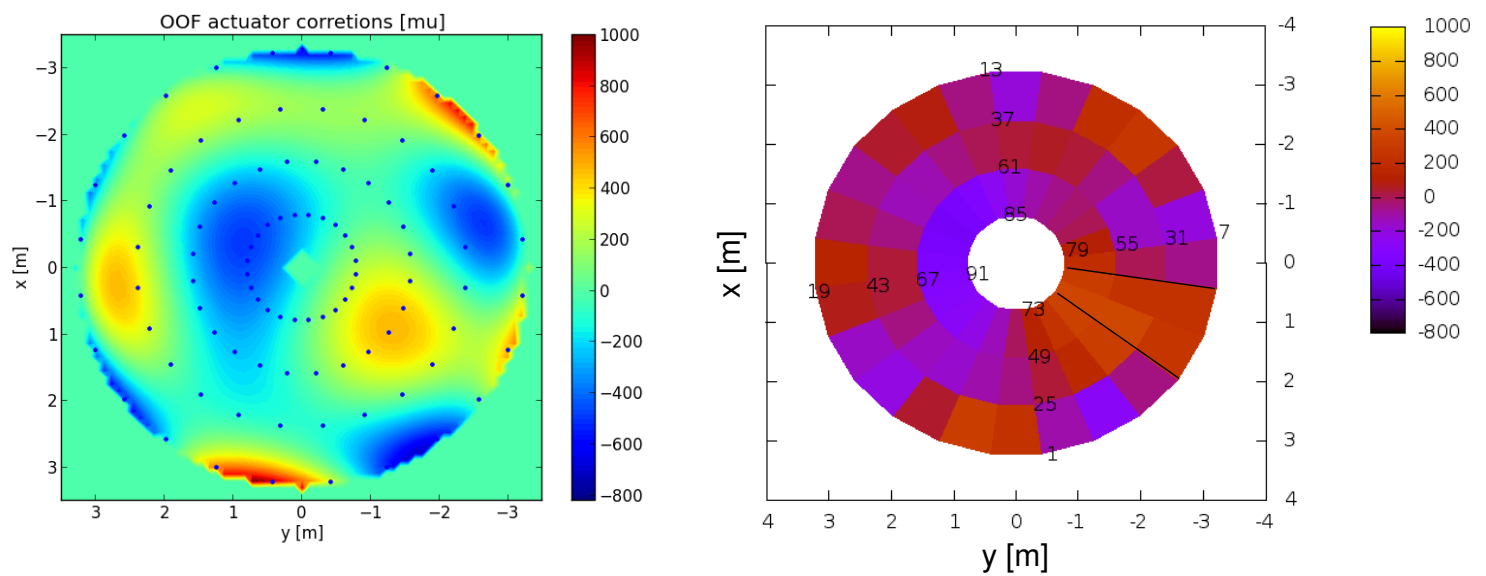

Figure 4: Left panel: Wave-front error map obtained from a measurement where a number of panels were lifted by $0.5 \mathrm{~mm}$. Right panel: Calculated actuator positions on a panel basis. The lifted panels are visible between the two lines. The measured shift agrees well with the introduced error.

based on the finite element model with real measurements and finally further reduce the elevation dependent gain loss.

\section{References}

[1] Nikolic, B.; Hills, R. E.; Richer, J. S., 2007, A\&A, 465, 679-683

[2] Nikolic, B.; Prestage, R. M.; Balser, D. S.; et al. 2007, A\&A, 685-693

[3] Bach, U.; Kraus, A.; Fürst, E.; Polatidis A., 2007, Effelsberg Memo Series (12-09-2007) 